\title{
Depresión y autoestima en adultos mayores institucionalizados y no institucionalizados en la ciudad de Lima
}

\author{
Carlos Orosco \\ Universidad de Lima, Perú
}

\begin{abstract}
Recibido: 15 de junio de 2015 / Aprobado: 7 de julio de 2015
\end{abstract}
El presente estudio se enfocó en los niveles de depresión y autoestima en los adultos mayores institucionalizados y no institucionalizados*. Las muestras estuvieron conformadas por 40 participantes sujetos institucionalizados y 45 no institucionalizados, los cuales fueron adultos mayores de estrato socioeconómico medio alto, con edades superiores a los 65 años. Se aplicaron el Inventario de Depresión de Beck - segunda versión (BDI-II) y el Inventario de Autoestima de Coopersmith (versión adultos). Por los resultados obtenidos se observó que existe una relación significativa e inversa entre la depresión y la autoestima en los grupos estudiados. Por otro lado, se encontró que al comparar los grupos con los niveles de depresión y autoestima estos presentan resultados similares.

depresión / autoestima / adulto mayor / institucionalizados / no institucionalizados

\section{Depression and Self-Esteem Among Institutionalized and Non-Institutionalized Senior Citizens in Lima}

The present study aimed at comparing levels of depression and self-esteem among institutionalized and non-institutionalized senior citizens. The respondents were 40 institutionalized and 45 non-institutionalized senior citizens both from the upper middleclass, and over 60 years of age. Both groups were given the Beck Depression Inventory (BDI) and the Coopersmith Self-Esteem Inventories-Adult form (CSEI). Results show that there is a significant and inverse correlation between depression and self-esteem. Additionally, both groups showed similar results on regarding levels of depression and self-esteem.

depression / self-esteem / seniors / institutionalization / non-institutionalization

* Se denomina 'institucionalizados' a los adultos mayores que residen en establecimientos de larga estadía, como casas de reposo, geriátricos, etc.; y 'no institucionalizados' a los ancianos que viven solos o con sus familiares.

Correo electrónico: cjorosco@ulima.edu.pe 


\section{INTRODUCCIÓN}

En la actualidad, se está produciendo un fenómeno poblacional en el mundo, tanto de crecimiento como de envejecimiento, dado que la tasa de nacimientos tiende a disminuir con respecto a las personas fallecidas, debido al cambio en la forma de vivir en la sociedad actual (Salvarezza, 1998). Además, en los datos señalados por el INEI la tasa bruta de natalidad entre los años 1995 al 2005, y con una proyección hacia el 2015 , tenderá a sufrir un decrecimiento considerable en el departamento de Lima, siendo esta disminución constante, con porcentajes de $19,3 \%$ en 1995 al $2000,17,7 \%$ en $2000-2005,16,4 \%$ en $2005-2010$ y $15,2 \%$ en $2010-2015$. Esto trae como consecuencia que el número de personas ancianas vaya incrementándose considerablemente en el transcurso de los años (INEI, 2007).

La vejez es un periodo crítico de la vida, que conlleva una serie de situaciones conflictivas, como la jubilación, la pérdida de seres queridos, la marcha de los hijos, las enfermedades crónicas, la proximidad de la muerte, entre otras, y a todo ello se le suman otros eventos negativos que ocurren casi inevitablemente. Estas circunstancias críticas producirán en los adultos mayores reacciones afectivas negativas, como la depresión (Fernández-Ballesteros, 2004).

Pero también se encuentra un grupo de adultos mayores que se sienten todavía en plenitud para realizar trabajos; sin embargo, por medio de la jubilación o de otras señales, la sociedad les dice que deben retirarse y dejar el puesto a gente más joven. Es decir, que la sociedad puede prescindir de ellos, lo cual lleva a que muchos adultos mayores no se sientan aceptados o pertenecientes a algo y a alguien, trayendo como consecuencia la baja autoestima (Ysern de Arce, 1999, citado por Salvarezza, 1998).

En ese sentido, la presente investigación busca dar a conocer a la población que la depresión en los adultos mayores es un factor de riesgo que puede afectar su autoestima. Asimismo, una baja autoestima también podría generar mayor depresión. La presencia de estas dos variables trata de advertir a la sociedad que no se encuentra debidamente preparada para hacer frente a esta problemática. Por ello, la finalidad de este estudio radica en determinar en qué medida se relacionan los niveles de depresión y autoestima en adultos mayores institucionalizados y no institucionalizados. Este propósito obedece a la necesidad de establecer que la depresión y la baja autoestima en el adulto mayor no son una consecuencia natural del envejecimiento sino más bien producto de la percepción negativa que tiene la sociedad sobre este grupo generacional.

Respecto al tema de estudio, Alcalá, Camacho y Giner (2007) llevaron a cabo un estudio en la ciudad de Sevilla, España, analizando si existen diferencias en la afectividad de la depresión de la tercera edad respecto a la depresión 
de la edad adulta. Aplicaron la escala PANAS-X a 120 sujetos (30 pacientes depresivos mayores de 65 años, 30 pacientes depresivos entre 25 y 50 años y dos grupos controles de 30 sujetos cada uno). Los resultados permiten establecer dos diferencias fundamentales de la depresión en la tercera edad frente a la depresión en el adulto: una menor intensidad de la afectividad negativa y una menor intensidad en los afectos de miedo, hostilidad y culpa. Además, se concluye que la tristeza no es un elemento diferenciador entre ambas poblaciones depresivas.

Canto y Castro (2004) realizaron un estudio en la ciudad de Yucatán, en México, en el cual estudiaron los niveles de depresión, ansiedad y autoestima en los ancianos, especialmente en quienes viven en estancias o que acuden a estas con frecuencia. De los resultados obtenidos se observó, de manera general, que hay probabilidades de que a mayor nivel de autoestima haya menor depresión; a mayor nivel de ansiedad, mayor probabilidad de depresión, y a menor autoestima, sea mayor el nivel de ansiedad y depresión. Al comparar las estancias con los asilos en cuanto a los niveles de depresión, ansiedad y autoestima, solo halló una diferencia significativa de mayores niveles de ansiedad en los asilos. Los niveles de depresión y de autoestima no resultaron significativos de estos lugares, aunque es común suponer que en ellos predominan la depresión y la baja autoestima.
Marín, Buendía y Ruiz-Hernández (2004), de la Universidad de Murcia, estudiaron las diferencias en la estructuración de la depresión en ancianos. Para ello, se ha trabajado con una muestra total de 507 adultos. De ellos 111 personas tenían una edad comprendida entre 20 y 30 años inclusive, 159 entre 35 y 55 , y las 237 restantes tenían una edad mayor o igual a la de 65 . Al conjunto de la muestra se le aplicó la escala CES-D, una entrevista clínica estructurada para la evaluación de trastornos depresivos según criterios DSM IV-TR y un inventario de síntomas de elaboración propia. Los resultados obtenidos arrojan diferencias en la configuración de los síntomas, tanto en el conjunto de la muestra como en el nivel subclínico. En el nivel clínico no se observan diferencias a lo largo del desarrollo adulto, si bien estas vuelven a aparecer en función de la edad, entre personas con elevada sintomatología que no reúnen los criterios clínicos de depresión.

\section{AdULTO MAYOR}

La vejez es un estado en la vida y el envejecimiento un proceso que sucede a lo largo del ciclo vital. Tanto la vejez como el envejecimiento humano son objetos de conocimiento multidisciplinares en el sentido de que el individuo humano es un ente bio-psico-social. Por esta razón, el individuo envejeciente o viejo es un sujeto de conocimiento psicológico (Fernández-Ballesteros, 2004). 
La tercera edad es un periodo crítico de la vida en el que se debe hacer frente a una serie de circunstancias personales, laborales, familiares y culturales, que modifican la percepción del sí mismo y afectan la propia identidad. La imagen que cada cual tiene de sí mismo comprende aspectos cognoscitivos y afectivos, y estos últimos estarían relacionados con la propia estima; esta ejerce una influencia universal sobre el modo de comportarse y la actitud ante la vida.

Por otra parte, el ingreso a una residencia geriátrica supone una forma de reubicación especialmente dura y difícil de elaborar. Cuando el anciano deja su casa para ir a vivir con algún familiar, sigue teniendo a su alcance personas que le conocen y que le recuerdan a cada gesto quién es, cómo es y quién fue. El conocimiento que familiares directos y amigos tienen sobre aspectos distintos de su biografía refuerza el reconocimiento de sí mismo, otorgando con esto una coherente continuidad a su identidad personal (Salvarezza, 1998).

Muchos adultos mayores llegan a la edad de la jubilación y se sienten todavía en plenitud para realizar sus trabajos. Aunque ven disminuidas sus potencialidades físicas al llegar a la vejez, sienten, sin embargo, que su mente sigue lúcida, y sus ganas de hacer las cosas permanecen inalteradas. En otras palabras, es como si se les dijera que podemos prescindir de ellos. Una de las primeras necesidades de todo ser humano es la de sentirse aceptado, querido, acogido, perteneciente a algo y a alguien, sentimientos en los que se basa la autoestima. La autoestima positiva es ser útil. Por lo tanto, no puede haber autoestima si el individuo percibe que los demás prescinden de él (Ysern de Arce 1999, citado en Salvarezza, 1998).

\section{Depresión en la terCera edAD}

La depresión es considerada uno de los trastornos de mayor complejidad en la población gerontológica, ya que puede tener efectos potencialmente nocivos en la salud de una persona mayor. A ello contribuyen una serie de factores, entre los que destacan las enfermedades somáticas, la disminución de las funciones cognitivas $\mathrm{y}$, al mismo tiempo, suele ser la causa más frecuente de sufrimiento emocional en la última etapa de la vida. Es importante añadir que el anciano tiende a no pedir ayuda especializada en centros de salud mental. El diagnóstico correcto de los problemas emocionales del anciano deprimido se convierte, por tanto, en una cuestión de extraordinaria importancia (Salvarezza, 1998).

La depresión puede ser mal diagnosticada en los ancianos debido a que los médicos atribuyen sus síntomas a enfermedades físicas o a que los consideran una parte normal del proceso de envejecimiento. Los síntomas de la depresión con frecuencia se traslapan con los cambios normales asociados con el envejecimiento, como pensamientos 
acerca de la muerte, cambios en el impulso o deseo sexual y en el patrón del sueño, y reducción de la energía. Puesto que la depresión acompaña algunas condiciones médicas como la enfermedad de Parkinson, apoplejía, trastornos tiroideos y ciertas deficiencias vitamínicas, identificar los síntomas puede ser una labor muy difícil (Papalia, 2009).

En referencia a lo planteado por Erikson, en la última etapa, llamada integridad del yo frente a la desesperación, afirma que mientras el individuo envejece y se jubila, tiende a disminuir su productividad, y explora la vida como personas jubiladas. Durante este periodo contempla sus logros y puede desarrollar integridad si considera que ha llevado una vida acertada. Si se ve la vida como improductiva, el adulto mayor se sentirá culpable por sus acciones pasadas, o si estima que no logró sus metas en la vida, se sentirá descontenta con esta, apareciendo la desesperación, que a menudo da lugar a la depresión (Papalia, 2009).

\section{LA AUTOESTIMA EN LA TERCERA EDAD}

La autoestima consiste en las actitudes del individuo hacia sí mismo. Cuando estas son positivas hablamos de buen nivel o alto nivel de autoestima. Al nombrar la palabra actitudes ya hemos incluido el mundo de los afectos y sentimientos y no solo el de los conocimientos, pues los componentes de la actitud encierran gran variedad de elementos psíquicos. De ahí que para la educación y formación de las personas, interesa mucho formar en actitudes porque así se puede asegurar una formación integral y no fraccionaria. Por lo mismo que las actitudes se encuentran integradas por factores cognitivos, afectivo-emotivos y conductuales, es muy difícil cambiarlas, pues radican en lo más profundo de la personalidad. Por eso también, un adecuado nivel de autoestima es garantía de que el sujeto podrá hacer frente con dignidad a importantes contrariedades de la vida; no decaerá su ánimo fácilmente (Bonet, 1994).

La modificación de las funciones sociales, ocasionadas por el acontecimiento que supone la jubilación, por la percepción de la vejez que tienen las propias personas ancianas y también por la percepción que se tiene de este nivel de edad por parte del resto de la sociedad, provocan que la consistencia interna de los diversos elementos del concepto de sí mismo resulte perturbada. Uno de los principales determinantes de la autoestima es la de etiquetarse a sí mismos como "viejo", debido a las connotaciones no positivas que este término conlleva en la actualidad (Salvarezza, 1998).

Ser viejo equivale a ser inútil, incapacitado, asexuado. Se estima que el adulto mayor pierde inevitablemente la mayoría de las capacidades de que gozaba en su vida anterior. No tiene otra capacidad. Es simplemente un discapacitado. Esta carga de prejuicios desemboca inevitablemente en ubicarlos en el único depósito con que cuenta la sociedad para ellos: los 
temidos cuarteles de invierno. Esto es, el lugar del paria, del marginado, del que ya no cuenta como integrante válido de la sociedad. En síntesis, la antesala de la muerte (Salvarezza, 1998).

En razón a todo lo presentado, no cabe duda de que la depresión y la autoestima son dos de las variables más importantes que afectan el comportamiento de las personas de la tercera edad, las cuales por lo general tienen dos condiciones básicas de vida: institucionalizados y no institucionalizados; por ello, en el presente trabajo se plantea la siguiente interrogante: ¿existe una relación significativa entre los niveles de depresión y autoestima en adultos mayores institucionalizados y no institucionalizados?

\section{HIPÓTESIS}

Para el desarrollo del estudio se plantean las siguientes hipótesis:

$\mathrm{H}_{1}$ : La depresión y la autoestima se relacionan de forma estadística significativa e inversa en los adultos mayores institucionalizados.

$\mathrm{H}_{2}$ : La depresión y la autoestima se relacionan de forma estadística significativa e inversa en los adultos mayores no institucionalizados.

$\mathrm{H}_{3}$ : La depresión y autoestima se diferencian en la intensidad de sus correlaciones en los adultos mayores institucionalizados respecto de sus pares no institucionalizados.
$\mathrm{H}_{4}$ : El grupo de adultos mayores institucionalizados presenta una mayor depresión que el grupo no institucionalizado.

$\mathrm{H}_{5}$ : El grupo de adultos mayores no institucionalizados presenta una mayor autoestima que el grupo institucionalizado.

\section{Método}

\section{Diseño de la investigación}

La investigación es de tipo correlacional, pues se recolecta información en ambas muestras tanto en institucionalizado y no institucionalizados, para determinar el grado de relación que existe entre la variable depresión y autoestima en la población de la tercera edad (Hernández, Fernández-Collado y Baptista, 2010). Adicionalmente, las muestras son comparadas, a fin de establecer la presencia de diferencias estadísticas significativas

\section{Participantes}

Se trabajó con una muestra de 85 sujetos (40 de ellos institucionalizados y el resto no institucionalizados). Los participantes fueron adultos mayores de estrato socioeconómico medio alto, comprendidos entre edades superiores a 65 años, dentro de la ciudad de Lima, de ambos sexos y que se encontraban institucionalizados o no institucionalizados. 


\section{Variables de estudio}

Se consideraron como variables relacionadas la depresión y la autoestima. Las variables de control son: adultos mayores de 65 años a más, los adultos mayores institucionalizados deben tener por lo menos 1 año de residencia en algún centro o asilo, los participantes deben residir en la ciudad de Lima.

\section{Instrumentos}

El Inventario de Depresión de Becksegunda versión (BDI-II) es un instrumento autoadministrado de 21 ítems. Cada ítem se califica en una escala de 4 puntos que va desde el 0 hasta el 3. Si un examinado hizo elecciones múltiples para un ítem, se utiliza la alternativa con el valor más alto. Se suman los 21 ítems y esto da una puntuación total. La puntuación máxima es 63 . Una puntuación de 0 a 3 indica un nivel de depresión mínimo, puntajes entre 14 y 19 un nivel leve, puntuaciones de 20 a 28 un nivel moderado y de 29 a 63 un nivel de depresión grave. En caso de que el evaluado puntúe 0 , este será interpretado como un nivel mínimo de depresión, así lo establecen los criterios de la prueba expuestos en líneas precedentes. En cambio, si la persona puntúa 63, dicho resultado será interpretado como la presencia de depresión grave (Beck, Rush, Shaw y Emery, 1993).

Está compuesto por ítems relacionados con síntomas depresivos, como la desesperanza e irritabilidad, cogniciones como culpa o sentimientos como estar siendo castigado, así como síntomas físicos relacionados con la depresión (por ejemplo, fatiga, pérdida de peso y de apetito sexual).

El Inventario de Autoestima de Coopersmith (versión adultos) fue construido por Stanley Coopersmith (1987), la versión adultos es una prueba diseñada para medir actitudes valorativas hacia el sí mismo dirigida a sujetos mayores de 15 años de edad y está compuesta por 25 reactivos. Los ítems se deben responder de acuerdo a si el sujeto se identifica o no con cada afirmación en términos afirmativos o negativos. Los ítems del inventario generan un puntaje total, así como puntajes separados en tres áreas: sí mismo general, social-pares y hogar. Sin embargo, estas tres áreas no han sido consideradas en el presente estudio debido a que los indicadores no daban puntuaciones significativas. Por ello, en el caso de este instrumento se decidió realizar una estimación general.

El puntaje máximo es de 100 y no existe escala de mentiras que invaliden la prueba. Los puntajes se obtienen sumando el número de ítems respondidos en forma correcta y multiplicando este por cuatro. Se considera de 0 a 24 nivel bajo, de 25 a 49 nivel medio bajo, de 50 a 74 nivel medio alto y de 75 a 100 nivel alto.

\section{Encuesta sociodemográfica}

Mediante esta encuesta se recolectó información personal de los adultos mayo- 
res. Las preguntas exploran los datos de filiación de los participantes, así como datos relacionados con su independencia para realizar tareas sin ayuda, satisfacción personal, solvencia económica, seguro médico, actividades de ocio, enfermedades padecidas, consumo de medicamentos, sentimientos de culpa en relación a hechos pasados, padecimiento de atrofias musculares, redes de soporte social, entre otros. Es importante mencionar que en el caso de las personas institucionalizadas la encuesta contaba con una pregunta específica en relación a la frecuencia de visitas por parte de sus familiares u otros allegados.

\section{Procedimiento de recolección de datos}

En primer lugar, se estableció cuáles serían los centros en los que se llevaría a cabo la aplicación de los cuestionarios. En la investigación se contó con la participación de cinco instituciones geriátricas, dos de las cuales pertenecían a órdenes religiosas.

Para recolectar los datos de la muestra se coordinó, con un mes de anticipación, con quienes tienen a su cargo el cuidado de las personas de la tercera edad que se encontraban institucionalizadas. En segundo lugar, se procedió a aplicar el Inventario de Depresión de Beck a los adultos mayores institucionalizados y a los no institucionalizados. En tercer lugar, se administró el Inventario de Autoestima de Coopersmith.

\section{Resultados}

\section{Contraste de hipótesis}

El contraste de la primera hipótesis realizado en adultos mayores institucionalizados en la tabla 1, posibilita observar que existe una correlación fuerte, negativa y significativa $(r=-0.60 \mathrm{p}<.05$ $\mathrm{r}^{2}=.36$ ). Este hallazgo permite concluir que la hipótesis 1 es válida.

El contraste de la segunda hipótesis realizado en adultos no institucionalizados en la tabla 1, posibilita notar que existe una correlación fuerte, negativa y significativa $\left(r=-.47 \mathrm{p}<.05 \mathrm{r}^{2}=.22\right)$. El hallazgo permite concluir que la hipótesis 2 es válida.

En lo que respecta a la tercera hipótesis, realizada a través del contraste de $\mathrm{Z}$ de correlaciones, los resultados presentados en la tabla 1 permite notar que no existen diferencias estadísticamente significativas $(\mathrm{Z}=0.83 \mathrm{p}>05)$ entre las correlaciones obtenidas en el grupo institucionalizado $\left(\mathrm{r}=-0.60 \mathrm{p}<.05 \mathrm{r}^{2}=.36\right)$ y el no institucionalizado $(\mathrm{r}=-0.47 \mathrm{p}<.05$ $\mathrm{r}^{2}=.22$ ), por lo que se concluye que la hipótesis 3 no es válida.

El contraste de la cuarta hipótesis, presentado en la tabla 2, permite apreciar que no existen diferencias estadísticas significativas en los niveles de Depresión $\left(\mathrm{X}_{(2, \mathrm{~N}=85)}^{2}=0.76 \mathrm{p}>.05\right)$ entre los niveles de depresión entre el grupo institucionalizado y el grupo no institucionalizado. Este hallazgo hace 
Tabla 1

Diferencias entre la correlación Depresión y Autoestima en adultos institucionalizados y no institucionalizados

\begin{tabular}{cccc}
\hline Variables & $\begin{array}{c}\text { Institucionalizado } \\
\mathrm{N}=40 \\
\text { Autoestima } \\
\mathrm{r}\end{array}$ & $\begin{array}{c}\text { Institucionalizado } \\
\mathrm{N}=45 \\
\text { Autoestima } \\
\mathrm{r}\end{array}$ & $\mathrm{Z}$ \\
\hline Depresión & $\begin{array}{c}0.60 * * \\
(.36)\end{array}$ & $\begin{array}{c}-0.47^{* * *} \\
(.22)\end{array}$ & \\
& & 0.83 \\
\hline
\end{tabular}

Nota: *** $\mathrm{p}<.001$, se aplicó la $\mathrm{r}^{2}$ como medida del tamaño del efecto y figura entre paréntesis.

posible concluir que ambos grupos presentan en los tres niveles de depresión valores equivalentes. Por lo tanto, la hipótesis 4 no es válida.

En lo que concierne al contraste de la hipótesis 5, presentado en la tabla 3, permite apreciar que no existen diferencias estadísticas significativas en los tres niveles de autoestima $\left(X^{2}(2, \mathrm{~N}=85)\right.$ $=3.09 \mathrm{p}>.05, \mathrm{~V}=.14)$ entre el grupo institucionalizado y el grupo no institucionalizado. Este hallazgo hace posible concluir que ambos grupos presentan niveles equivalentes de autoestima, por lo que la hipótesis 5 no es válida.

\section{Discusión}

Respecto a la primera hipótesis específica, la cual propone que existe una relación significativa e inversa entre la depresión y la autoestima en un grupo de ancianos institucionalizados, los hallazgos permiten concluir que es válida (véase la tabla 2): es decir que a medida que los adultos mayores presentan ma-

Tabla 2

Análisis comparativo del nivel de depresión por tipo de grupo

\begin{tabular}{lcccc}
\hline \multirow{2}{*}{ Grupo de comparación } & \multicolumn{3}{c}{ Nivel de depresión } & \multirow{2}{*}{ Total } \\
\cline { 2 - 4 } & Ausencia & Leve & Moderada & \\
\hline Institucionalizado & 16 & 15 & 9 & 40 \\
No institucionalizado & 21 & 17 & 7 & 45 \\
\hline \multicolumn{1}{c}{ Total } & 37 & 32 & 16 & 85 \\
$\mathrm{X}^{2}{ }_{(2, \mathrm{~N}=85)}=0.76 \mathrm{P}=.684$ & & & & \\
$\mathrm{~V}$ de Cramer $=.07$ & & & &
\end{tabular}


yor autoestima tienden a presentar menor depresión y viceversa. Este resultado concuerda con los hallazgos reportados por Canto y Castro (2004), quienes estudiaron los niveles de depresión, ansiedad y autoestima en los ancianos, en la ciudad de Yucatán, México, especialmente en quienes viven o acuden a estancias con frecuencia, encontrando una correlación similar a la obtenida en el estudio entre el nivel de autoestima y los niveles de depresión.

En el análisis de la segunda hipótesis específica, en la cual se establece que existe una relación significativa e inversa entre la depresión y la autoestima en un grupo de adultos mayores no institucionalizados, los resultados permitieron concluir que también es válida (véase la tabla 3). Este hallazgo coincide con el comunicado por Canto y Castro (2004), el cual reveló que los ancianos que viven en asilos o acuden a estancias con frecuencia presentan una correlación similar entre el nivel de autoestima y los niveles de depre- sión. Para tratar de explicar el presente hallazgo se puede citar a Furstenberg (1989), quien ha demostrado que existe una correlación entre el autoconcepto en la edad y las medidas de salud mental, pues refiere que la gente que se describe a sí misma como anciana tiende a presentar menos satisfacción de vida, más baja moral y más bajas medidas de salud psicológica. Además, este resultado concuerda con lo mencionado por Zung (1967), Bibring (1953) y otros, debido a que el bajo autoconcepto es el principal factor de la depresión en la tercera edad. Por su parte, Busse (1954) indica que encuentra diferencias entre sujetos adultos y ancianos en cuanto al mecanismo de los episodios depresivos, de tal manera que en los ancianos se encuentra una relación entre el fenómeno depresivo y el bajo autoconcepto.

En lo que concierne a la tercera hipótesis específica vinculada con las diferencias en la relación que existe entre la depresión y la autoestima en un grupo de adultos mayores institucionalizados

Tabla 3

Análisis comparativo del nivel de la autoestima por tipo de grupo

\begin{tabular}{ccccc}
\hline \multirow{2}{*}{ Grupo de comparación } & \multicolumn{3}{c}{ Nivel de autoestima } & \multirow{2}{*}{ Total } \\
\cline { 2 - 4 } & Bajo & Medio bajo & Medio alto & \\
\hline Institucionalizado & 25 & 12 & 3 & 40 \\
No institucionalizado & 29 & 8 & 8 & 45 \\
\hline Total & 54 & 20 & 11 & 85 \\
\hline
\end{tabular}

$\mathrm{X} 2(2, \mathrm{~N}=85)=3.09 \mathrm{p}=.214$

$\mathrm{V}$ de Cramer $=.14$ 
con respecto a los no institucionalizados, los hallazgos permiten concluir que esta no es válida (véase la tabla 1). Este resultado concuerda con lo reportado por Canto y Castro (2004), quienes al comparar las estancias con los asilos en cuanto a los niveles de depresión y autoestima, encontraron que la relación entre estas variables no resultó significativa. Es decir que la depresión y la autoestima serían independientes del lugar donde residen los adultos mayores. Lo cual permite afirmar que no por encontrarse residiendo en un centro geriátrico necesariamente presentarán una mayor depresión y menor autoestima, lo cual podría generalizarse a los adultos mayores que residen con sus familiares o solos. Este hallazgo concuerda con lo mencionado por Buttler y Lewis (1973), los cuales atribuyen la mayoría de los sentimientos negativos hacia los ancianos a las actitudes sociales, debido a que sufren discriminación, y este resultado de apartarlos puede generar depresión, y la baja autoestima puede ser el producto de la pérdida de un lugar definido en la sociedad. Es decir que la posible causa de la depresión y la baja autoestima en adultos mayores podrían deberse a la percepción negativa que tiene de ellos la sociedad. Los hallazgos se contraponen con lo indicado por Thoits (1985) y Krause (1987), quienes establecen que en la vejez se rompen muchos de los vínculos sociales como consecuencia, de la jubilación, la pérdida de seres queridos y la institucionalización. Por ello consideran que la autoestima de los ancianos institucionalizados en comparación con el de aquellos que continúan viviendo en el hogar familiar se va a caracterizar por un mayor descenso de la autoestima y una mayor degradación, lo cual no se observa en el presente estudio.

En lo referente a la cuarta hipótesis específica, en la cual se establece que el grupo de adultos mayores institucionalizados presenta un mayor de nivel depresión que el grupo no institucionalizado, los hallazgos permiten concluir que no es válida (véase la tabla 2), lo cual se corresponde con los resultados obtenidos por Canto y Castro (2004), en cuyo estudio se aprecia que no existen diferencias significativas en los niveles de depresión entre los grupos participantes. Este hallazgo concuerda por lo indicado por Salvarezza (1998), quien menciona que el proceso de envejecimiento no siempre es placentero, debido a que el adulto mayor se ve vulnerable a trastornos médicos crónicos y debilitantes, la pérdida de amigos y de seres queridos, y la incapacidad para participar en actividades que antes disfrutaba, puede resultar una carga muy pesada para el bienestar emocional de una persona que está envejeciendo, estos y otros aspectos producen emociones negativas que pueden explicar la frecuencia de la depresión en este grupo.

En lo que concierne al análisis de la quinta hipótesis específica, en la cual se establece que el grupo de adultos mayores no institucionalizados presenta un mayor nivel de autoestima 
que el grupo institucionalizado, como se puede apreciar según lo evidenciado en la tabla 3, no es válida. Es decir, no existen diferencias estadísticas significativas en el nivel de la autoestima entre el grupo institucionalizado y el grupo no institucionalizado. Este resultado corresponde con lo indicado por Bonet (1994), quien refiere que el adulto mayor, aunque se sienta saludable, se desconcierta ante dos experiencias contradictorias: por un lado se siente bien y con ganas de trabajar, pero por otro lado la sociedad le expresa que son prescindibles, lo cual representa un duro golpe a la autoestima.

\section{Conclusiones}

1) Existe una relación estadísticamente significativa e inversa entre la depresión y la autoestima en los grupos estudiados, es decir, tanto en los adultos mayores institucionalizados como en sus pares no institucionalizados, encontramos que a mayor depresión se infiere menor autoestima.

2) Existe una relación estadísticamente significativa e inversa entre la depresión y la autoestima en un grupo de adultos mayores no institucionalizados, es decir que a medida que presentan mayor autoestima tienden a presentar menor depresión, y viceversa.

3) No existen diferencias estadísticas significativas en la relación entre la depresión y la autoestima en un grupo de adultos mayores institu- cionalizados con respecto a los no institucionalizados, es decir que al comparar los grupos con los niveles de depresión y autoestima estos presentan resultados similares.

4) El grupo de adultos mayores institucionalizados no presenta mayores niveles de depresión que el grupo de no institucionalizados, es decir que no se obtienen diferencias estadísticas significativas en la comparación de los niveles de depresión entre los grupos participantes.

5) El grupo de adultos mayores no institucionalizados no presenta una mayor autoestima que el grupo de no institucionalizados, es decir que no existen diferencias estadísticas significativas en los niveles de la autoestima entre el grupo institucionalizado y el grupo no institucionalizado.

\section{RECOMENDACIONES}

Promover el desarrollo de investigaciones similares sobre otras temáticas importantes del comportamiento que afectan a los adultos mayores, tales como resiliencia, tipo de envejecimiento, consecuencias psicológicas de las enfermedades crónicas, redes de soporte social, autoeficacia, etc., con la finalidad de generar aportes teóricos y servir como base empírica a partir de la cual se pueden desarrollar intervenciones y estrategias preventivas futuras, a fin de mantener la calidad de vida de este grupo poblacional; y del 
mismo modo, contribuir en la reflexión y estudios de la problemática del adulto mayor en el contexto nacional.

Promover el desarrollo de programas comunitarios integrales, el apoyo familiar, la evitación de internamientos innecesarios; de talleres de aprendizaje, de memoria, expresivos, reflexivos, de multiestimulación, etc., y otras actividades como medio de prevención de futuras patologías, como es el caso de la depresión y la baja autoestima en los adultos mayores. Además, fomentar el envejecimiento activo como proceso de optimización de las oportunidades de salud, participación social, realización personal, planificación de la vejez y seguridad, con el fin de mejorar la calidad de vida a medida que las personas envejecen; es decir, las intervenciones que crean ambientes de ayuda y fomentan opciones saludables, y que sean relevantes para la autonomía, la independencia y la interdependencia social.

Elaborar un perfil del adulto mayor en el cual se tenga presente la variedad de cambios (jubilación, viudez, menos ingresos económicos y decadencia física) a los que se enfrentan conforme van transcurriendo los años. El desarrollo de este perfil será beneficioso para los profesionales, puesto que se determinarán las áreas necesarias de trabajo con el adulto mayor. Todo ello con la finalidad de desarrollar y ampliar el campo de acción de la psicología en relación con esta población en particular.
Finalmente, construir instrumentos especializados para la población de adultos mayores que tomen en cuenta sus limitaciones funcionales y sus particularidades en relación con el aspecto social, emocional, familiar, entre otros. Asimismo, cuidar que sean instrumentos confiables y con una adecuada validez.

\section{RefERENCIAS}

Alcalá, V., Camacho, M., y Giner J. (2007). Afectos y depresión en la tercera edad. Psicothema, 19(1), 49-56. Recuperado de http://www.psicothe$\mathrm{ma} \cdot \mathrm{com} / \mathrm{psicothema} \cdot \mathrm{asp}$ ?id=3327

Beck, A., Rush, A., Shaw, B., y Emery, G. (1993). Terapia cognitiva de la depresión. Bilbao: Desclée de Brower.

Beck, A., Steer, R., A., y Brown, G. K. (1996). BDI-II. Beck Depression Inventory II-. (2. ${ }^{\mathrm{a}}$ ed.). San Antonio, TX: The Psychological Corporation.

Bibring, E. (1953). The mechanism of Depression. En P. Greenacre (Ed.), Affective Disorders (pp. 14-47). New York: International University Press. (Trad. castellana: Perturbaciones de la Afectividad. Buenos Aires: Hormé).

Bonet, J. V. (1994). Sé amigo de ti mismo. Manual de autoestima. Madrid: Sal Terrae.

Busse, E. W., Barnes, R. H., y Silverman, A. J (1954). Studies of the processes of aging: factors the influence the psyche of elderly persons. Ame- 
rican Journal of Psychiatry, 110, 897-903.

Buttler, R., y Lewis, M. (1973). Aging and Mental Health: Positive Psychosocial Approaches. En Robson (1988). Self-esteem: A psychiatric View. British Journal of Psychiatry, 153, 6-15.

Canto, G., y Castro, E. (2004). Depresión, autoestima y ansiedad en la tercera edad: un estudio comparativo. Enseñanza e Investigación en Psicología, 9, 257-270. Recuperado de http://redalyc.uaemex.mx/redalyc/src/inicio/ ArtPdfRed.jsp?iCve=29290204

Coopersmith, S. (1987). Self-esteem inventory adult form. San Francisco, CA: Freeman.

Fernández-Ballesteros, R. (2004). La psicología de la vejez. Encuentros multidisciplinares, 16, 1-11. Recuperado de http://www.encuentros multidisplinares.org

Furstenberg, A. L. (1989). Older people's age self concept. Social casework. The Journal of Contemporary Social Work, 70, 268-275.

Hernández, R., Fernández-Collado, C., y Baptista, L. (2010). Metodología de la investigación (5. ${ }^{\text {a ed.). }}$ México, D. F.: McGraw-Hill.

INEI - Instituto Nacional de Estadística e Informática. (2007). Recuperado de http://www.inei.gob.pe
Krause, N. (1987a). Chronic financial strain, social support, and depressive symptoms among older adults. Psychology and Again, 2, 185-192.

Marín, R., Buendía Vidal, V., y RuizHernández, A. (2004). Estructura diferencial de la depresión en ancianos. Psiquis, 25(3), 108-121. Recuperado de http://www.psiquis.com/ art/04_25_n03_A03.pdf

Nunnally, J., y Bernstein, I. (1995). Teoría psicométrica. México, D. F.: McGraw-Hill.

OMS - Organización Mundial de la Salud. (2000). Clasificación de los trastornos mentales y del comportamiento, con glosario y criterios diagnósticos de investigación. Madrid: Medica Panamericana.

Papalia, D. (2009). Desarrollo del adulto $y$ vejez. (3. ${ }^{\mathrm{a}}$ ed.). México, D. F.: McGraw-Hill.

Salvarezza, L. (1998). Una mirada gerontológica actual. Buenos Aires: Paidós.

Thoits, P. A. (1985). Social support as coping assistance. Journal of Consulting and Clinical Psychology, 54, 416-423.

Zung, W. K. (1967). Factor influencing the self-rating depression scale. Archives of General Psychology, 16, 543-547. 